\title{
Selection of Mathematical Problems in Accordance with Student's Learning Style
}

To the level of engineering by using an expert system

\author{
Elena Fabiola Ruiz Ledesma \\ Escuela Superior de Cómputo \\ Instituto Politécnico Nacional \\ México
}

\author{
Juan J. Gutiérrez García \\ Escuela Superior de Cómputo \\ Instituto Politécnico Nacional \\ México
}

\begin{abstract}
This article describes the implementation and development of an expert system as a support tool to tackle mathematical topics, by using Bayesian networks as engine of inference and a learning styles, as well as the difficulty level of problems and establish the base of the classifier probabilistic. The expert system makes decisions as which element to visualize at a specific moment, gives the student the best resource, and supervises the user progress. The article is divided into three sections, the first one deals with the construction of the expert system, the second one presents the operation of the system through the classification of students consistent with their profile, which is based on the prevailing learning style among them and in the difficulty level that problems have so that the student reaches in solving successfully. It also shows the operability of the system in respect of the allocation of digital resources in accordance with the identified profile and gradually provides more assignments with different difficulty levels, as the student progresses. An experimental study was performed by means of which the system was assessed under 30 students to the level of engineering and those who studied the Applied Calculus course in their second semester of the degree course. This group was named the study group (SG). The SG used the system for one semester. The results at the initial and final evaluation were from 3.58 to 7.37 for CG and SG respectively. Applying the F test, a statistically significant difference in increase was found (p $<0.002$ ). These results showed that SG identified the concept of derivative and applied that concept correctly in real problems solving correctly $\mathbf{7 4 \%}$ of the final questionnaire, so it is concluded that the system expert opens a new way in educational research.
\end{abstract}

Keywords—technology; research projects; education; learning

\section{INTRODUCTION}

Learning based on traditional computing systems and mobile devices converge in its use in what is called Mobile Learning also known as M-learning, by allowing users to stay connected to the learning environment, learning resources, members of the educational community, such as teachers and students, no matter where they are [1].

Thus, the learning process is no longer bounded to a specific location and will depend essentially of the student's willingness to accede to learning resources. The problem occurs when the student does not have enough incentive to find out the available content offered by some current systems, either because they have very complex exercises, or on the contrary, they have very simple ones, or due to they do not have a variety of activities that help them to understand that concepts as these are presented in only one form for all students, even though each of them may have different styles of learning, as noted by Felder and Silverman [2].

The applications of the learning systems are quite extensive, there are several e-learning platforms that allow to structure content based on a large variety of multimedia resources, however, they present each element in static way, they do not make decisions or determine which element visualizes in a certain moment; they do not check which is the most suitable. Furthermore, they do not oversee adequately the progress of the user. This fact supports the study of areas such as artificial intelligence applied to learning within the framework of e-learning, since in several contexts its efficient functioning has been demonstrated [3].

The field of expert systems (also associated with systems of knowledge representation or knowledge engineering) are an important part of the field of Artificial Intelligence. Fundamentally, they represent the knowledge of the expert in the domain (area of expertise), Being applied, including as a tool by the very same expert [4].

As part of the research reported in this article, a survey was carried out on a sample of $50 \%$ of the students from the Escuela Superior de Cómputo (ESCOM), an Academic Unit of the Instituto Politécnico Nacional (IPN), and there were obtained the following results:

1) Generally, students are used to the e-learning as well as the m-learning,

2) More than $80 \%$ of the sample considers that an online application, which supports learning and it could help in their learning process,

3) $90 \%$ of the students expressed their willingness to find out more about the use of mobile devices in the learning process.

4) More than $80 \%$ of the students have failed a basic training course (Mathematics or Physics) and they consider that it would be very useful to have a website that allows them t1o solve problems and practice what they have learned in class, as this would allow them to face better the exams they solve at their on-site courses.

In this study we also found that students know some sites on the Internet that offer resources either to reinforce 
knowledge, such as videos that explain problem solving, or to practice what they learn, through exercises to solve, but point out that it does not adapt to their level since the problems are of equal degree of difficulty for all the users and they think that they would solve exercises and easy problems in the beginning and that the degree of complexity is increasing. They also feel that they would feel more relaxed if there is a supervision of what they do to be able to review their progress.

The present work intends to develop a support tool for the teaching of some topics that are worked in the Calculus, using Bayesian networks as the engine of inference and for the determination of the profiles of the students will be considered the registers of semiotic representation [5], which will be the basis of the probabilistic classifier

\section{B. Expert Systems}

An expert system can be defined as a computer system that simulates human experts in a given area of expertise. As such, an expert system should be able to process and store information, learn and reason in deterministic and uncertain situations and communicate with users and / or other expert systems, make appropriate decisions, and explain why they have taken such decisions.

This kind of systems encode a knowledge base and reasoning rules to determine or conclude the solution of a particular problem. Are formed by various interrelated parts: a rule base, a base of facts, an inference engine and an user interface [6].

The knowledge of the expert is represented by the rule base that are generally of the form $R_{i}: P_{r}(x) \Rightarrow C(x)$, where $P_{r}(x)$ it's a premise and $C(x)$ a conclusion. The conditions of application of a rule are the premises and new knowledge are conclusions.

\section{a) Inference engine: Bayesian networks}

The inference engines used rules in various ways, in particular the method of reasoning will be based on probabilistic classification with Bayesian networks. This method is characterized by a multivariable representation of data to be processed, which allows to describe complex relationships of certain elements and nonlinear, they represent causal relationships, thus allowing handling uncertainty in events unobserved [7].

A Bayesian network represents a joint probability distribution on a 4-tuple $\left(G, f_{x} Q, \Theta\right)$, where:

- $\left(G, f_{x} Q\right)$ it's a causal network.

- $G$ it's an acyclic digraph.

- The set $x$ of nodes $G$ its defined by $\left\{x_{i} \mid i \leq n\right\}$ of random variables with $r$ possible states and $\Theta$ it's a set $\left\{\Theta_{i} \mid i \leq n\right\}$.

So, the expansion of the joint distribution is:

$$
P\left(Y \mid f_{1}, \ldots, f_{N}\right)=\prod_{i=1}^{N} p\left(f_{i} \mid Y\right) \cdot P(Y)
$$

For the posterior probability of how it can be the status of variables in a Bayesian network, Bayes' theorem is used.

\section{b) Classifiers probabilistic as an artificial learning}

Learning from the perspective of Artificial Intelligence, it is considered as a process of induction of knowledge that allows us to generalize behaviors from an unstructured information provided in the form of examples incorporating design workable solutions to problems through the study of the computational complexity of these.

The computational analysis and performance of machine learning algorithms is a branch of statistics known as Computational learning theory. Different learning algorithms are grouped into a taxonomy based on the output thereof. Some types of algorithms are:

Supervised learning. The algorithm produces a function that establishes a correspondence between inputs and desired outputs of the system. An example of this type of algorithm is the classification problem, where the learning system tries to assign a label or classify a series of vectors using one of several categories (classes). The knowledge base system consists of labeled examples above. This type of learning can be very useful in biological research problems, Computational Biology and Bioinformatics.

Unsupervised learning. The entire process of modeling is performed on a set of examples formed only by system inputs. There is no information on the categories of those examples. Therefore, in this case, the system must be able to recognize patterns to assign labels to new entries.

Semi supervised learning. This type of algorithms combine the above two algorithms to classify properly. Marked and unmarked data is taken into account.

Reinforcement learning. The algorithm learns observing the world around him. Your input information is the feedback you get from the outside world in response to their actions. Therefore, the system learns based on trial and error.

Transduction. Similar to supervised learning, but not explicitly constructed a function. Tries to predict future categories of examples based on input examples, their respective categories and examples new system.

Multitasking learning. Learning methods using knowledge previously learned by the system face to face similar problems to those already seen

\section{c) Registers semiotic representation}

Semiotic representations are representations that use signs, they can expressed in natural language or algebraic, graphs or figures geometric. These semiotic representations are the means through which a person can externalize their mental representations in order to make them visible or accessible to others. The ability to change the records of semiotic representation is necessary in the mathematics learning and the importance of coordinating the different registers of semiotic representation. Many difficulties experienced by students can be described and explained as a lack of coordination between representation registers, in particular graphic, numeric and algebraic [5]. 


\section{Methodology}

The expert system has several components, modules and persistent entities. Optimization problems are working and interacting with students through a virtual tutor who tells the procedure to be followed at all times. As shown in Figure 1, the student begins to access the pretest module, where it presents a diagnostic test that, through various reagents, assesses student skills and deficiencies [6].

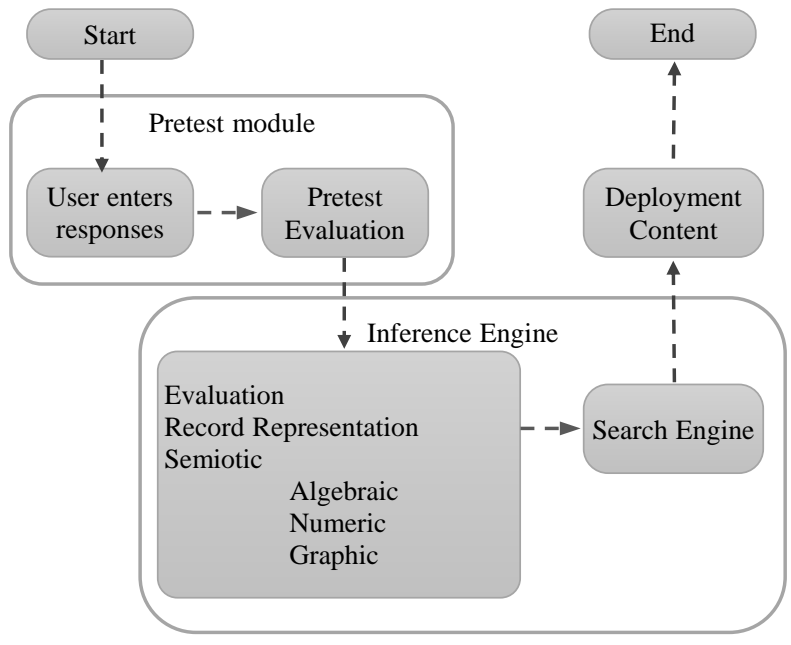

Fig. 1. Flow user action with the expert system

Figure 2 shows an example of the graphical user interface (GUI) provided to the student to work with the pretest. This interface provides the user with the following elements: the problem statement, a descriptive picture, different options to choose in response to problem and mechanisms of recurrent asynchronous storage

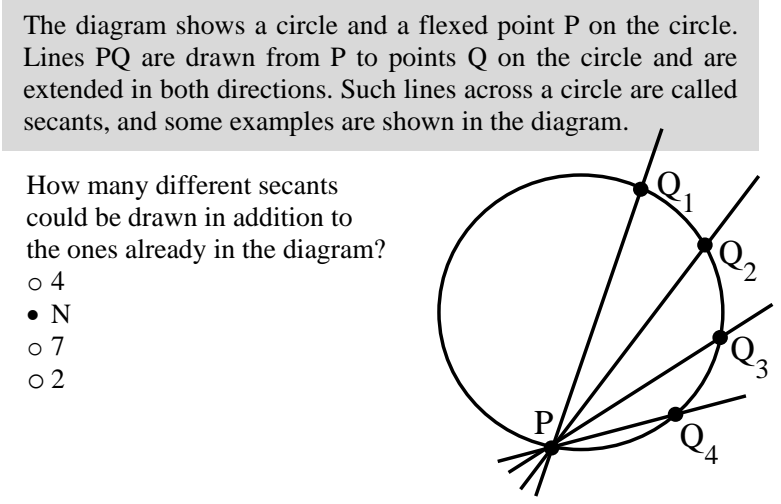

Fig. 2. GUI pretest for a record graphical representation

To determine which register of semiotic representation has the highest efficiency is assigned a score according to the complexity of the reagent. The evaluation is carried out in the inference engine using probabilistic classification based on Bayesian networks [7].

The search engine takes the result of the inference drawn and creates a data structure ramified from a collection of associative structures that link resources, activities and teaching materials. This collection is stored in a database. Finally, an exhaustive search is performed to determine the optimal route learning, that is to say, the set of suggested activities for the student.

\section{A. System Overview}

The expert system consists of a set of applications and Web services that engage in the following modules:

Users. In this module, the user has the ability to create your account, manage your profile, change personal data and set your preferences. This module is responsible for controlling the restricted access to the system.

Control of students. It allows to monitor student progress by monitoring system access and viewing activity logs and pretests presented. It provides the user with the role of teacher the ability to generate reports and statistics showing the student's behavior on the platform.

Administration. The user administrator role is the only one with access to this module. It allows management of secondary modules and specific components of search engine and inference.

Management pretests. Since this module creating, editing, and deleting query evaluation tests for the student is managed. The role of teacher has the privileges to access this module.

Content management. Enables management activities, resources and learning materials enabling the creation and editing content paths. These routes will be used by the search engine to determine the learning path that suits the profile of each student. The inference engine is responsible for composing the profile based on the records of semiotic representation.

Activity logs and backups. This module has tools that allow the export of records stored in the database through the scheduling of full or partial backups in plain text with SQL format.

\section{RESULTS}

We worked with a group of 30 college students, who started their Calculus course. This group was called the study group (SG). These students enrolled in the system and solved a questionnaire, which allowed them to determine their preference for the representation register, which they were used to work. Once they were placed in one of three profiles: Algebraic, numerical, and graphic, the system allocated resources to each student, according to their profile, in order to make the students feel comfortable resolving the problems. Once the activities were resolved, the students would upload the work developed as an attachment and in the system selected the response presented in subparagraphs. Subsequently, the system provides students with examples of solved problems, through simulations, using the three registers, graph, numerical and algebraic.

So, that the student can review how to obtain the same result using different semiotic register. About the part of digital educational resources there is also a league that re-addresses some program that allows the student to construct his or her own simulation and knowledge. 
Finally, the system gives new problems to be solved by the student, offering him options of records to employ in its resolution. Figures 3 and 4 show the resolution of a rate of change problem using the algebraic register.

A village is situated 20 kilometers from a straight railroad track that passes through village A. Determine the position relative to B in which a train station $C$ must be built on the railroad track so that the journey from village $\mathrm{A}$ to village $\mathrm{B}$, making the trip by rail and $\mathrm{CB}$ by road, as long as possible, if the speed by rail is $80 \mathrm{Km} / \mathrm{h}$ and the road speed is $20 \mathrm{Km} / \mathrm{h}$.

Algebraic procedure:

1. Find the time as a function of the distance $\mathrm{x}$ from the station to the point A.

$$
\begin{aligned}
& v=\frac{e}{t} \Rightarrow t=\frac{e}{v} \\
& t_{1}=\frac{d}{20}=\frac{\sqrt{20^{2}+(a-x)^{2}}}{20} \quad t_{2}=\frac{x}{80}
\end{aligned}
$$

where: $\mathrm{d}$ is the distance elapsed at a time $\mathrm{t}_{1} \mathrm{y} \mathrm{x}$ is the distances elapsed by the railway at a time $t_{2}$

2. Total time spent.

3. Function to optimize

$$
t=\frac{\sqrt{20^{2}+(a-x)^{2}}}{20}+\frac{x}{80}
$$

4. Critical points

$$
t(x)=\frac{\sqrt{20^{2}+(a-x)^{2}}}{20}+\frac{x}{80}, x \in[0, a]
$$

$$
\begin{aligned}
t^{\prime}(x)=\frac{-(a-x)}{20 \sqrt{20^{2}+(a-x)^{2}}}+\frac{1}{80} \\
t^{\prime}(x)=0 \Rightarrow-80(a-x)+20 \sqrt{20^{2}+(a-x)^{2}}=0 \\
4(a-x)=\sqrt{20^{2}+(a-x)^{2}} \\
16(a-x)^{2}=20^{2}+(a-x)^{2} \\
15(a-x)^{2}=20^{2} \\
a-x=\frac{20}{\sqrt{15}} \Rightarrow x_{0}=a-\frac{20}{\sqrt{15}}
\end{aligned}
$$

Fig. 3. Rate of change problem using the algebraic register

$$
\begin{aligned}
& \text { 5. Discussion } \\
& \begin{aligned}
\square \quad & \text { to } x=0, t=\frac{\sqrt{20^{2}+a^{2}}}{20} \\
\square \quad \text { to } x & =a, t_{2}=1+\frac{a}{80} \\
\square \quad \text { to } x & =a-\frac{20}{\sqrt{15}}, t_{3}=\frac{\sqrt{20^{2}+\frac{20^{2}}{15}}}{20}+\frac{a-\frac{20^{2}}{15}}{8}
\end{aligned}
\end{aligned}
$$

Fig. 4. Rate of change problem using the algebraic register

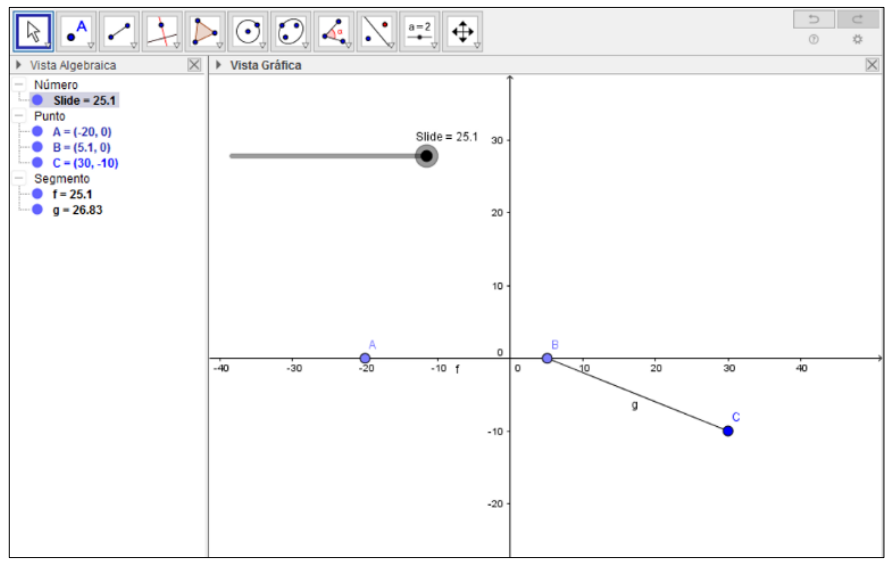

Fig. 5. Rate of change problem using the graphics register
The figures 5 and 6 show the construction of this problem using a dynamic geometry program.

\section{Procedure in GeoGebra $\odot$ :}

1) With the straight-line tool take the option of segment and a length. Locate point A of coordinates $(0,-20)$.

2) Determine the slides by giving values, minimum 0 and maximum 25.15.

3) Make another line segment from point B to point $\mathrm{C}(30,-20)$

4) Built another line segment from point B to point D $(30,0)$.

5) Use the slider to produce animation.

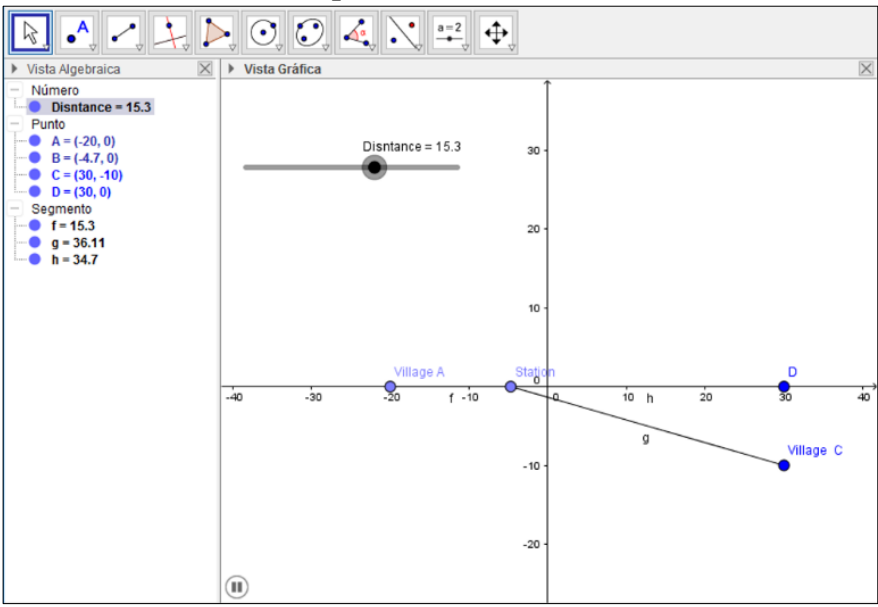

Fig. 6. Rate of change problem using the graphics register

The figure 7 and 8 show an example of numerical representation register that is related with the same concept rate of change.

A flow of water falls into a tank at a rate of constant change, such that for each unit that increases over time, the water depth increases by two units. The chart and graph illustrate this situation.

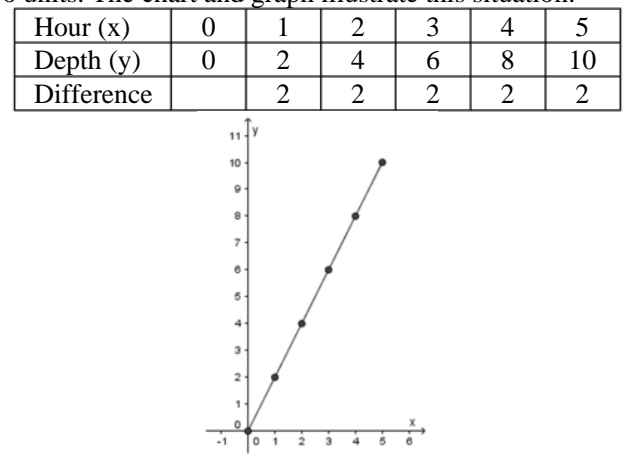

What is the rate of change of depth with respect to time if $x=$

Fig. 7. Rate of change problem using the numerical register

The study group (SG) used the system for one semester.

Once the students of the study group (SG) worked with the concepts of derivative and rate of change, by solving various activities and problems, using the expert system, they solved a final questionnaire, which asked them concepts applied to different situations of the same matter of Calculus. When 
obtaining the average of the group in this questionnaire it was found that in relation to the initial had a significant increase since of having obtained 3.58, increased to 7.37. The F test was used and the increase was found to be ( $\mathrm{p}<0.002)$.

The table shows the amount of litter dumped into the sea at certain
times of the year
\begin{tabular}{|l|c|c|c|c|c|c|c|}
\hline Day (x) & 1 & 2 & 3 & 4 & 5 & 6 & 7 \\
\hline Tons (y) & 0.25 & 1.55 & 2.85 & 4.15 & 5.45 & 6.75 & 8.05 \\
\hline Difference & & 1.3 & 1.3 & 1.3 & 1.3 & 1.3 & 1.3 \\
\hline
\end{tabular}

What is the rate of change of the amount of garbage with respect to the day if $x=$ Thursday (day 5)?

Fig. 8. Rate of change problem using the numerical register

This result showed that SG identified the concept of derivative and correctly applied that concept to real problems solving correctly $74 \%$ of the final questionnaire, so it is concluded that the system expert open a new way in educational research.

\section{CONCLUSIONS}

Using probabilistic classifiers for the implementation of inference engines in an expert system allows a multivariate representation of the data to be treated, which in turn shows a description of complex relationships of certain elements, also considering the handling of uncertainty. When using semiotic (graphic, numerical, iconic and algebraic) registers as primary classification elements, an appropriate form of presenting the aspects related to the learning paths of the area to be reinforced in the student is parameterized.

\section{ACKNOWLEDGMENT}

We are grateful for the support provided to IPN's SIP (research project 20164801. We also thank COFAA.

\section{REFERENCES}

[1] Kurbel K., Hilker, J., (2002). Requirements for a mobile e-Learning Platform, IASTED Intl. Conf. on Commun., Internet and Information Technology, US Virgin Islands.

[2] Felder, R and Silverman, L. (1988) "Learning and Teaching Styles," Journal of Engineering Education, Vol. 78, No.7, pp. 674-681.

[3] Micarelli, A., Stamper J., and. Panourgia, K. (2016). Intelligent Tutoring Systems. Sun, T., Liu H. (2013).

[4] Henderson, H. (2003). Encyclopedia of computer science and technology. New York, NY: Facts on File.

[5] Duval, R. (1998). Registros de representación semiótica y funcionamiento cognitivo del pensamiento. Investigaciones en Matemática Educativa II, p. 173-201.

[6] Sun, T., Liu H. (2013). Design of Fault Diagnosis Expert System of Transformer. AMM, vol. 291-294, pp. 2557-2561, 2013.

[7] Woolf, B. (2009). Building intelligent interactive tutors. Amsterdam: Morgan Kaufmann .

[8] Russell, S., Norving, P. (2009). Artificial Intelligence: A Modern Approach, 3rd. Prentice Hall, Englewood Cliffs. 